\title{
The Mindful Attention Awareness Scale for Adolescents (MAAS-A): Psychometric Properties in a Dutch Sample
}

\author{
Esther I. de Bruin • Bonne J. H. Zijlstra • \\ Eva van de Weijer-Bergsma • Susan M. Bögels
}

Published online: 28 June 2011

(C) The Author(s) 2011. This article is published with open access at Springerlink.com

\begin{abstract}
The factor structure and psychometric properties of the Dutch version of the Mindful Attention Awareness Scale for Adolescents (MAAS-A) was studied in a sample of adolescents ( $n=717$; age range, $11-17$ years) of the general population. The MAAS-A and other questionnaires measuring other constructs were administered in high schools across the Netherlands. A one-factor structure was demonstrated using principal component analysis and was further confirmed using confirmatory factor analysis. The MAAS-A was shown to have high internal consistency. Expected negative correlations between mindfulness and self-reported stress and emotion regulation strategies such as rumination and catastrophizing were found. Further, mindfulness was positively correlated with happiness, healthy self-regulation, and with another recently developed measure of mindfulness in children and adolescents, the Child and Adolescent Mindfulness Measure. Mindfulness as measured by the MAAS-A correlated positively with quality of life, but an expected positive relationship with acceptance was not found. Interestingly, adolescents without meditation experience scored higher on the MAAS-A than adolescents without this experience. Further, adolescents with chronic disorders scored lower on the MAAS-A than adolescents without these disorders. Overall, this study has shown evidence of the first valid and reliable Dutch measure of mindfulness for adolescents. The factor structure, internal consistency, and convergent and divergent
\end{abstract}

E. I. de Bruin $(\bowtie) \cdot$ B. J. H. Zijlstra • E. van de Weijer-Bergsma • S. M. Bögels

Faculty of Behavioral and Social Sciences, Research Institute of Child Development and Education, University of Amsterdam, Nieuwe Prinsengracht 130,

1018 VZ, Amsterdam, the Netherlands

e-mail: e.i.debruin@uva.nl validity as well as their relationship to quality of life are comparable to the original MAAS-A.

Keywords MAAS-A · Mindfulness · Internal consistency · Construct validity. Psychometric properties

\section{Introduction}

Adolescent mental disorders occur very frequently and lead to enormously high societal costs. According to the NIMH, the lifetime prevalence of a mental health disorder for 1318 -year-olds is $46.3 \%$ and just over $20 \%$ of these adolescents suffer from a severe mental disorder. Lifetime prevalence for this age group is estimated to be $9 \%$ for attention deficit hyperactivity disorder (ADHD), 14\% for mood disorders, and 25\% for anxiety disorders (Merikangas et al. 2010). Mental health care costs for children and adolescents in the USA are up to around nine billion dollars a year (NIMH statistics 2006). For the Netherlands, a costof-illness (COI) study showed that a yearly 697 million euros was spend on mental health disorders in 0-19-yearolds, and costs increased with age. Adolescent mental health disorders were allocated the largest part of this sum (e.g., 37\% for 15-19-year-olds; Poos et al. 2008).

In a meta-analysis of psychotherapeutic treatment for youth depression, a mean small to medium effect size (ES) of .34 was demonstrated (Weisz et al. 2006). Another systematic review of the effectiveness of psychotherapies for depression in adolescents as compared to treatment as usual or no treatment showed that psychotherapies (particularly cognitive behavioral therapy and interpersonal therapy) had superior effects over the other treatments, but this did not last. After 5 months, the effectiveness of psychotherapy was no longer significant (Watanabe et al. 
2007). Further, Davis et al. (2011) recently showed that many treatments for anxiety disorders in children and adolescents still need to be established as efficacious. In addition, long-term effects of cognitive behavioral treatments for youth with ADHD are limited and generalization effects are small (Pelham and Fabiano 2008). It thus seems that in the treatment of high-cost and high-prevalence adolescent mental health disorders, additional psychological interventions might be beneficial.

One of these relatively new interventions is mindfulnessbased treatment. Mindfulness is defined as paying attention in a particular way: on purpose, in the present moment, and nonjudgmentally (Kabat-Zinn 1994). Mindfulness-based treatments are extremely popular and are still rapidly increasing worldwide. In the USA alone, mindfulness-based treatments are applied in over 250 health care centers (Jha et al. 2007). Mindfulness is originally based on Eastern (Buddhist) meditation practices (i.e., Baer 2003). Through the practice of meditation, an ability to direct one's attention can be developed. A main goal of mindfulness-based interventions is the moment-to-moment perception of events with full awareness without distortion from associated thoughts from the observer (Kabat-Zinn et al. 1985). Mindfulness-based trainings differ from the conventional (cognitive) behavioral therapies in that they focus on universal difficulties rather than on specific problems (Bögels et al. 2008).

Although several (meta-analytic reviews of) randomized studies emphasize the efficacy of mindfulness-based interventions in adults (e.g., Baer 2003; Grossman et al. 2004; Hofmann et al. 2010; Segal et al. 2002), studies of mindfulness training in adolescents are still in its infancy (Burke 2009; Greco and Hayes 2008). It has been suggested however that the level of mindfulness moderates psychological functioning in adolescents (Marks et al. 2010). Thus far, only a few, small, not randomized, no control group studies have been published. However, preliminary findings are positive; reductions in stress, improvements in sleep, improvements in ADHD symptoms, and aggression in adolescents who suffer from substance abuse, sleep problems, ADHD, and conduct disorder (CD) have been reported (Bögels et al. 2008; Bootzin and Stevens 2005; Singh et al. 2007; Zylowska et al. 2008). In a review of efficacy of meditations in youngsters from 6 to 18 years, it was found that sitting meditation was effective in the treatment of physiological conditions (i.e., high blood pressure; ES ranged from .16 to .29) and much more so in the treatment of psychological/psychiatric conditions (i.e., ADHD, anxiety; ES ranged from .27 to .70; Black et al. 2009).

Only one randomized clinical trial of mindfulness-based stress reduction (MBSR) as an adjunct to treatment as usual (TAU) versus TAU alone is reported with 14-18-year-old adolescent psychiatric outpatients $(n=102$; Biegel et al.
2009). Nearly $50 \%$ of the adolescents suffered from mood disorders, and anxiety disorders were present in $30 \%$ of the participants. On self-report measures, decreases in anxiety and stress at post-treatment and at 3-month follow-up were much larger for the MBSR + TAU group (ES from .70 to .89) than in the TAU only group (ES from -.01 to .16). Further, DSM-IV classifications and Global Assessment of Functioning scores showed large improvements in the MBSR + TAU condition, whereas hardly any diagnostic change occurred in the TAU only condition. Diagnostic change was expressed as the reduction in number of DSM-IV classifications after treatment. For the MBSR+TAU group, nearly $50 \%$ showed this reduction in number of classifications whereas in the TAU only group, practically no diagnostic change occurred (Biegel et al. 2009). The largest decrease in classifications applied to the mood disorders.

Further, our research group carried out a pilot study in 14 adolescents with severe externalizing disorders. After combined mindfulness training for adolescents and their parents, parents reported direct and longer-term improvement in adolescents' externalizing and attention problems $(\mathrm{ES}=1.1)$, self-control, and better attunement to others, and adolescents themselves reported large improvements on their own goals and symptoms (Bögels et al. 2008). In another pilot study of our research group in 10 adolescents with ADHD, it was found that adolescents showed improved performance on two computerized sustained attention tests, directly after training and at follow-up (ES ranged from .80 to 1.8 ) in addition to improvement in externalizing and attention problems (ES ranged from .60 to 1.8 ), as reported by adolescents, fathers, and teachers (Van de Weijer-Bergsma et al. 2011).

Although mindfulness-based interventions seem to be promising as an adjunct psychological training for adolescents, valid and reliable mindfulness questionnaires are lacking, all the more so for adolescents. Mindfulness questionnaires with good psychometric properties are needed to determine whether mindfulness skills do indeed increase after participation in a mindfulness-based intervention in clinical and non-clinical populations, and to assess whether this increase mediates the subsequent decrease in psychological or psychiatric symptoms (e.g., Baer et al. 2006; Bishop et al. 2004). Therefore, the aim of the present study is to assess the psychometric properties of the Dutch translation of the recently adapted adolescent version of the Mindful Attention Awareness Scale for Adolescents (MAAS-A; Brown et al. 2011).

The MAAS (Brown and Ryan 2003) was originally developed for adults from normative and clinical populations and is worldwide one of the most commonly used mindfulness questionnaires. Development of the MAAS was completed with help of (Buddhist) teachers and highly trained students of mindfulness, and the authors of the 
MAAS consider mindfulness to primarily be a quality of attention and define the concept as "a receptive state of attention that, informed by an awareness of present experience, simply observes what is taking place" (Brown and Cordon 2009; Brown and Ryan 2003; Brown et al. 2007). In the MAAS, mindfulness is considered to be a one-dimensional construct, different from, for instance, the five mindfulness facets described in the Five Facet Mindfulness Questionnaire (Baer et al. 2006).

Summarized, the aim of our study is to assess several psychometric properties of the Dutch MAAS-A in a large sample of adolescents from the general community and to compare these findings with its (original) counterpart in the USA. We will assess factor structure and internal consistencies, as well as construct validity, which will be examined by calculating correlations with constructs such as rumination, catastrophizing and stress (divergent validity), and happiness, healthy self-regulation, acceptance, and correlations with another mindfulness questionnaire specifically developed for children and adolescents (convergent validity). Further, the relationship between mindfulness and quality of life is assessed. We tested the following hypotheses. First, based on studies of the original MAAS-A (Brown et al. 2011), we expected the Dutch version of the MAAS-A to consist of a single factor. Second, since mindfulness-based interventions have been shown to have positive effects on psychological wellbeing in adolescents (Biegel et al. 2009), we expected a positive relation between mindfulness and the psychosocial and physical aspects of quality of life. Third, we expected the MAAS-A to show a positive correlation with the Child and Adolescent Mindfulness Measure (CAMM; Greco et al. 2011), recently developed to measure mindfulness in children and adolescents. Since both instruments tap on the overall construct of mindfulness, but place an emphasis on different aspects (i.e., awareness versus non-judgmental acceptance), we expected a high but not perfect correlation. Fourth, we expected mindfulness to correlate positively with happiness and negatively with rumination, catastrophizing, and stress since previous studies have shown the stressreducing effect of mindfulness-based interventions and the resulting improvements in quality of life and happiness (i.e., Baer 2003). Healthy self-regulation and acceptance are ingredients of most mindfulness-based interventions, and we therefore expected these constructs to correlate positively with the MAAS-A. In addition, it was previously shown that the adult MAAS correlated with self-regulation (Brown and Ryan 2003), and we therefore expected the MAAS-A to do so as well. Last, we expected adolescents with meditation and/or yoga experience to report higher levels of mindfulness than adolescents without this experience, and we expected adolescents who suffer from a chronic disease to be less mindful than adolescents who did not suffer from chronic diseases.

\section{Methods}

Participants and Procedure

The sample consisted of $n=781$ high school students from two high schools in the Netherlands, one in the most urban area and the other in a more rural area. High school principals have given oral and written permission for participation of their school in this project. Subsequently, parents of the adolescents were informed via letters, emails, and information brochures about the validation project and adolescents whose parents refused to participate were excluded from further study $(n=24 ; 3 \%)$. Also, at the time of testing, adolescents who refused participation themselves were excluded $(n=8 ; 1 \%)$. Teachers of the different participating classes were informed by the school principals. The procedure was approved by the Institutional Review Board.

The questionnaires were administered anonymously in the classroom (approximately $30 \mathrm{~min}$ ) and two of the project members were present to supervise the administration and to assist the adolescents where necessary. The set of questionnaires was administered to $n=749$ adolescents. Participants who did not fill out the questionnaires, for whom more than $10 \%$ of the items were missing or whose reports were considered unreliable (i.e., consistently rated the highest or the lowest scores on all items), were excluded from the analyses $(n=32 ; 4 \%)$. Data of $n=717$ adolescents were included in the analyses (age, $M=14.6 ; \mathrm{SD}=1.5$; range, $11.4-17.9 ; 51.3 \%$ male). Males and females did not differ in MAAS-A scores, $t(715)=0.24 ; p>.05$. When less than $10 \%$ of items were missing, item ratings were coded as the average of the other items of the scale. The education level of our sample was high $38.5 \%$ mixed high school classes HAVO/VWO/gymnasium, 32.1\% HAVO, 29.4\% $\mathrm{VWO} /$ gymnasium). In the Netherlands, $\mathrm{VWO}$ /gymnasium is the highest level of high school, pre-university education (duration 6 years) and HAVO is the level below that, senior general secondary education (duration 5 years), and in the first 1 to 3 years of high school, these levels are combined. Age and education were correlated with MAAS-A scores $(r=-0.22 ; p<.001$ and $F$ $(2.71)=9.14 ; p<0.001$, respectively), both separately and combined, therefore partial correlations with other constructs will be calculated. The majority of adolescents were of Dutch origin $(96.5 \%)$.

\section{Measures}

\section{$M A A S-A$}

The MAAS-A (Brown et al. 2011) consists of 14 items that measure the level of mindfulness (example items are "It 
seems 'I'm running on automatic', without much awareness of what I'm doing", or "I tend not to notice feelings of physical tension or discomfort until they really grab my attention"). The items are answered on a six-point scale $(1=$ Almost always; $6=$ Almost never $)$ on which higher scores are an indication of higher trait mindfulness. The MAAS-A is derived from its adult counterpart the MAAS, which is one of the most commonly used mindfulness questionnaires (Brown and Ryan 2003). The MAAS has been validated in various samples of students $(\alpha=.82)$ and adults from the general community $(\alpha=.87)$ (Brown and Ryan 2003; MacKillop and Anderson 2007). A one-dimensional factor structure of the MAAS was shown. Test-retest reliability was good (intraclass correlation=.81). In a large number of samples, Brown and Ryan (2003) clearly showed indications of good convergent, discriminant, and incremental validity. The MAAS correlated with various measures of well-being. The MAAS was related to lower neuroticism, depression, anxiety, and unpleasant affect, and to life satisfaction, optimism, and self-esteem.

Subsequently, Brown and Ryan (2003) showed that the MAAS differentiated significantly (effect size .50) between Zen meditators $(n=50)$ and adults from the general community $(n=50)$. In addition, the MAAS was validated for a population of patients with cancer $(n=122)$. Again, a one-dimensional structure was found and internal consistency was high $(\alpha=.87)$. Lower levels of mindfulness were related to lower levels of psychological well-being in the cancer patients as well as in the adults from the general community (Carlson and Brown 2005).

Very recently, the MAAS-A has been validated in adolescent normative $(n=595)$ and psychiatric populations $(n=102)$ (Brown et al. 2011). Exploratory factor analysis (EFA) showed a one-factor structure which was confirmed by confirmatory factor analysis (CFA). Cronbach's alpha was good (varied from .82 to .84 ). Test-retest reliability was examined in a subsample $(n=131)$ and was high (intraclass correlation=.79). The MAAS-A, like its adult counterpart, correlated with psychological well-being and healthy self-regulation. When psychiatric adolescent patients (14-18 years) were randomly divided over MBSR and TAU, it was found that the MBSR participants showed a significant increase in MAAS-A scores from pretest to follow-up, whereas participants in the TAU condition did not show these improvements. The authors conclude that the MAAS-A is sensitive to the effects of mindfulness training (Brown et al. 2011).

The authors of the MAAS-A granted us permission for translation and back translation of the MAAS-A, and authorized our final version, which is partly based on and adjusted from a previous Dutch translation of the adult MAAS (Schroevers et al. 2008).

\section{Subjective Happiness Scale}

The Subjective Happiness Scale (SHS) is a measure of happiness (Lyubomirsky and Lepper 1999) and consists of four items that are rated on a seven-point scale (i.e., "In general I consider myself"; $1=$ Not a very happy person; $7=$ A very happy person). Item 4 is reverse scored, and all items load on one factor (Lyubomirsky and Lepper 1999). In general, the SHS has been shown to have high internal consistency ( $\alpha$ varied between .74 and .94 in different studies). Different psychometric properties of the SHS such as test-retest reliability and divergent and convergent reliability have all shown good results (i.e., Lyubomirsky and Lepper 1999; Tkach and Lyubomirsky 2006). Internal consistency for this studies sample was acceptable $(\alpha=.74)$.

\section{Pediatric Quality of Life Inventory Scale}

Adolescents' quality of life was assessed with the Pediatric Quality of Life Inventory Scale (PedsQL) (Varni et al. 1999). The PedsQL is a 23 -item five-point questionnaire measuring quality of life in four domains: physical, emotional, social, and school functioning (i.e., "I feel afraid or scared", or "I have low energy") and has been shown to have good reliability ( $\alpha$ varied from .80 to .90 ) and validity properties (Varni et al. 2001). A psychosocial health summary score is composed of the items of the emotional, social, and school functioning subscales.

The PedsQL reliably distinguishes healthy children from pediatric patients. The reliability and validity of the Dutch PedsQL are satisfactory (Bastiaansen et al. 2004). In this study, three scores are included in the analyses: physical functioning ( $\alpha=.77)$, psychosocial health $(\alpha=.77)$, and total scale score $(\alpha=.86)$.

\section{Healthy Self-Regulation Subscale}

The Healthy Self-Regulation Subscale (HSR) (West 2008) measures self-regulation skills of adolescents (i.e., "I recognize when I'm upset and calm myself"), and the 12 items are rated on a six-point scale. The reliable HSR is derived from the larger Mindful Thinking and Action Scale for Adolescents (West et al. 2008). Preliminary evidence shows that the HSR is sensitive to the effect of mindfulness training in adolescents (West and Kram 2008), and the HSR was also included in the validation study of the original MAAS-A (Brown et al. 2011). Good internal consistency for this studies sample $(\alpha=.80)$ was shown.

\section{Childrens's Acceptance and Mindfulness Measure}

The Children's Acceptance and Mindfulness Measure (CAMM) (Greco et al. 2011) is a recently developed 
questionnaire that measures mindfulness in children and adolescents (i.e., "I keep myself busy so I don't notice my thoughts or feelings") and the 10 items are rated on a five-point scale. The CAMM has been shown to be reliable $(\alpha=.81)$, and positive correlations have been shown with quality of life, social skills, and academic performance. Negative correlations were shown with somatic complaints, internalizing and externalizing symptoms (Greco et al. 2011). Internal consistency in our adolescent sample was good $(\alpha=.80)$.

\section{Stress Questionnaire for Youth}

The Stress Questionnaire for Youth (Stress Vragenlijst Voor Kinderen; SVK) (Hartong et al. 2003) assesses the level of experienced stress in children and adolescents. The 19-item questionnaire is rated on a four-point scale (i.e., "I often feel relaxed"). Internal consistency has been shown to be good (Meijer et al. 2008), and in the current adolescent sample, reliability of the SVK was also good $(\alpha=.82)$.

\section{Cognitive Emotion Regulation Questionnaire}

The Cognitive Emotion Regulation Questionnaire (CERQ) (Garnefski et al. 2001) measures nine different coping styles and consists of 36 items (i.e., "I think I have to accept that this has happened" [scale acceptance] or "I often think that what I have experienced is much worse than what others have experienced" [scale catastrophizing]). The items are rated on a five-point scale. Cronbach's alpha for the nine different scales varies from good to very good (Garnefski et al. 2001). For this study, only scales with a theoretical relationship with mindfulness were included. Internal consistencies in the current sample for these three scales were good ( $\alpha$ was .70, .76, and .73 for rumination, catastrophizing, and acceptance, respectively). Rumination refers to the tendency to repeatedly think about feelings and thoughts that are associated with negative events. Catastrophizing refers to repeatedly re-occurring thoughts about how terrible the event has been, and acceptance refers to thoughts in which one accepts what has happened.

\section{Statistical Analyses}

In accordance with the original MAAS-A analyses (Brown et al. 2011), our sample was split into two groups, stratified by age and education level. Sample A $(n=364)$ was used to perform EFA, and sample B $(n=353)$ was used to perform CFA. Internal consistency (Cronbach's alpha) was examined. Pearson correlations with mental health-related constructs were calculated to examine construct validity. Pearson correlations were also calculated between the MAAS-A and the other mindfulness measure, the CAMM.
To assess the relationship between meditation or yoga experience and the MAAS-A, adolescents were dichotomized on a categorical variable (meditation/yoga experience or not). The two groups were compared using an independent samples $t$ test. Further analyses compared the adolescents with regular experience to the previously mentioned groups. Similar analyses were carried out to assess the relationship between chronic disorders (i.e., asthma, epilepsy, migraines) and the MAAS-A.

\section{Results}

\section{EFA}

To examine whether the Dutch version of the MAAS-A also consists of a single factor, a principal component analysis (PCA) was conducted. The Kaiser-Meyer-Olkin [KMO] measure of sampling adequacy was .91. Two factors emerged with an eigenvalue above 1 (5.06 and 1.03, respectively). Since the difference between the eigenvalue of the first component and the following components is so large and the second component contained many negative and small (in absolute sense) loadings, a single-factor solution seems to be most appropriate. The factor loadings for the single-factor principal component solution of the MAAS-A items are presented in Table 1.

It can be seen that all item loadings easily exceeded the critical value of .30 (Hair et al. 1995) and ranged from .37 for item 1 to .75 for item 13 .

\section{CFA}

A CFA was conducted using LISREL 8.8 on sample B. Because inspection of the data showed that a normal distribution did not apply, robust maximum likelihood estimation was used. The fit of the covariance matrix as implied by the single-factor model was close to the observed (asymptotic) covariance matrix: RMSEA $=.035$, $90 \%$ CI $(.019, .050)$, comparative fit index $=.991$, parsimony normed fit index $=.822$, even though, as expected, the single-factor model did not completely fit the observed (asymptotic) covariance matrix, $\chi^{2}(77)=$ $189.97 ; p<.001$. The items were all significantly $(p<.001)$ related to the latent scale.

\section{Internal Consistency}

Internal consistency based on 14 items for the adolescent sample was good $(\alpha=.86$ for sample $\mathrm{A}$ and .85 for sample B). Further, item-total correlations varied between .34 (item 1) and .65 (item 13). The average item scores and the MAAS-A total score can be seen in Table 2. 
Table 1 Factor loadings of Dutch MAAS-A items $(n=364)$ and original MAAS-A items

\begin{tabular}{lcc}
\hline Items & $\begin{array}{c}\text { Original } \\
\text { MAAS-A }\end{array}$ & .37 \\
MAAS-A & .48 & .47 \\
\hline 1. I could be experiencing some emotion and not be conscious of it until some time later. & .63 & .55 \\
2. I break or spill things because of carelessness, not paying attention, or thinking of something else. & .72 \\
3. I find it difficult to stay focused on what is happening in the present. & .40 \\
4. I tend to walk quickly to get where I'm going without paying attention to what I experience along the way. & .28 \\
5. I tend not to notice feelings of physical tension or discomfort until they really grab my attention. & .76 \\
6. I forget a person's name almost as soon as I have been told it for the first time. & .64 \\
7. It seems I am "running on automatic," without much awareness of what I am doing. & .47 \\
8. I rush through activities without being really attentive to them. & .67 \\
9. I get so focused on the goal I want to achieve that I lose touch with what I am doing right now to get there. & .53 \\
10. I do jobs or tasks automatically, without being aware of what I am doing. & .47 \\
11. I find myself listening to someone with 1 ear, doing something else at the same time. & .32 \\
12. I find myself preoccupied with the future or the past. & .63 \\
13. I find myself doing things without paying attention. & .59 \\
14. I snack without being aware that I am eating. & .62 \\
\hline
\end{tabular}

${ }^{\text {a }}$ Original MAAS-A factor loading are copied from manuscript by Brown et al. (2011)

\section{Construct Validity}

To investigate the construct validity of the MAAS-A, partial correlations with measures of stress, rumination, and catastrophizing were calculated (divergent validity) as well as correlations with measures of acceptance, happiness, healthy self-regulation, and another measure of mindfulness (convergent validity). All correlations between mindfulness and these other constructs can be seen in Table 3. The partial Pearson correlations have been controlled for age and educational level. All correlations in Table 3 are significant at the $p<.001$ level, except for the correlations of Acceptance (zero-order: $p=.77$, partial $p=.59$ ).

As predicted, mindfulness correlated negatively with stress $(r=-.54 ; p<.001)$, rumination $(r=-.27 ; p<.001)$, and catastrophizing $(r=-.34 ; p<.001)$. The more mindful an adolescent reported to be, the less stress he/she perceived in his/her daily life and the less use he/she made of emotion regulation strategies such as rumination and catastrophizing. Further, as can be seen in Table 3, being more mindful was related to more happiness $(r=.33 ; p<.001)$ and to more healthy self-regulation strategies such as being able to stop

Table 2 Means (SD) of the Dutch MAAS-A items and total score in adolescent sample $(n=717)$

Items

$M(\mathrm{SD})$ Dutch

MAAS-A

1. I could be experiencing some emotion and not be conscious of it until some time later

$4.39(1.27)$

2. I break or spill things because of carelessness, not paying attention, or thinking of something else.

$4.66(1.36)$

3. I find it difficult to stay focused on what is happening in the present.

$4.40(1.32)$

4. I tend to walk quickly to get where I am going without paying attention to what I experience along the way.

$4.05(1.48)$

5. I tend not to notice feelings of physical tension or discomfort until they really grab my attention.

$4.45(1.37)$

6. I forget a person's name almost as soon as I've been told it for the first time.

$4.70(1.49)$

7. It seems I am "running on automatic," without much awareness of what I am doing.

$4.26(1.34)$

8. I rush through activities without being really attentive to them.

$4.49(1.18)$

9. I get so focused on the goal I want to achieve that I lose touch with what I am doing right now to get there.

$4.58(1.27)$

10. I do jobs or tasks automatically, without being aware of what I am doing.

$4.56(1.27)$

11. I find myself listening to someone with 1 ear, doing something else at the same time.

$4.05(1.36)$

12. I find myself preoccupied with the future or the past.

$3.70(1.52)$

13. I find myself doing things without paying attention.

$4.53(1.22)$

14. I snack without being aware that I am eating.

$4.90(1.37)$

MAAS-A total score

61.73 (11.09) 
Table 3 Partial correlations between Dutch MAAS-A and other constructs (controlled for age and educational level)

\begin{tabular}{lll}
\hline Construct & $\begin{array}{l}\text { Zero-order } \\
\text { correlation }\end{array}$ & $\begin{array}{l}\text { Partial } \\
\text { correlation }\end{array}$ \\
\hline Stress (SVK) & $-.56^{* * *}$ & $-.54 * * *$ \\
Rumination (CERQ) & $-.28^{* * *}$ & $-.27^{* * *}$ \\
Catastrophizing (CERQ) & $-.35^{* * *}$ & $-.34 * * *$ \\
Acceptance (CERQ) & -.01 & -.02 \\
Happiness (SHS) & $.34 * * *$ & $.33^{* * *}$ \\
Healthy self-regulation (HSR) & $.50^{* * *}$ & $.48^{* * *}$ \\
Quality of life (PedsQL) & & $.30^{* * *}$ \\
Physical functioning & $.30^{* * *}$ & $.50^{* * *}$ \\
Psychosocial health & $.52^{* * *}$ & $.48^{* * *}$ \\
Total scale score & $.50^{* * *}$ & $.54 * * *$ \\
Mindfulness (CAMM) & $.55^{* * *}$ & \\
\hline
\end{tabular}

CAMM Child and Adolescent Mindfulness Measure, CERQ Cognitive Emotion Regulation Questionnaire, HSR Healthy Self-Regulation, PedsQL Pediatric Quality of Life Inventory Scale, SHS Subjective Happiness Scale, SVK Stress Vragenlijst voor Kinderen (Stress Questionnaire for Youth)

$* * * p \leq .001$

oneself from saying mean things $(r=.48 ; p<.001)$. Contrary to our expectation, mindfulness was not positively correlated with acceptance $(r=-.02 ; p>.05)$. As expected, the total score on the PedsQL was significantly positively related to total MAAS-A score $(r=48 ; p<.001)$. A higher quality of life was positively related to mindfulness. This finding applied to both the physical domain $(r=.30 ; p<.001)$ and more strongly to the psychosocial domain $(r=.50$; $p<.001)$. Last, as hypothesized, a positive correlation between the CAMM and the total MAAS-A score was found $(r=.54 ; p<.001)$.

\section{Mindfulness (MAAS-A) and Meditation/Yoga Experience}

Around $10 \%(n=73)$ of the adolescents reported to have some experience with meditation and/or yoga. Within this group, a further $22 \%(n=16)$ reported to have regular (daily or at least weekly) experience. Significant differences were found between the adolescents with and without meditation/yoga experience, $t(715)=-2.81 ; p=.005$. Surprisingly, it was the adolescents without meditation/yoga experience who scored higher on the MAAS-A-NL total. Within the group with some experience with meditation and/or yoga, there was no further difference with those who had regular experience, $t(69)=.99 ; p=.33$. The observed mean for those with regular experience (61.31) was close to mean of those that had no experience (61.98). This difference was also not significant $(p=.81)$. Since mindfulness was also measured with the CAMM, we compared groups on the CAMM as well, and results pointed in the same direction with adolescents with meditation experience rating lower on mindfulness, $t(714)=-2.40 ; p=.016$.

Mindfulness (MAAS-A) and Chronic Disorders

Around $12 \%(n=84)$ of the adolescents reported to suffer from a chronic disorder, of which asthma occurred most often $(n=32)$ followed by severe headaches or migraine $(n=12)$. Significant differences were found between adolescents with and without chronic disorders, $t(705)=$ $-3.28 ; p=.001$. Adolescents with a chronic disorder indicated to be less mindful than adolescents without a chronic disorder. Again, findings were similar on the CAMM, $t(704)=-2.87 ; p=.004$.

\section{Discussion}

The aim of this study was to assess different psychometric properties of one of the first officially translated Dutch adolescent mindfulness questionnaires, the MAAS-A. We examined the internal consistency, the factor structure, and the construct validity in a large sample of adolescents from the general population and compared findings to results from the original MAAS-A.

Several conclusions can be drawn from this study. First, results of the PCA showed that the Dutch version of the MAAS-A has the same one-factor structure as the original MAAS-A. The CFA clearly supported this. The internal consistency of the Dutch MAAS-A was good, and we can therefore conclude the Dutch version of the MAAS-A is a reliable instrument.

Second, the relationship between mindfulness and quality of life was positive as expected. Higher self-reports of mindfulness were related to better functioning in the physical and social domains of life. This is in accordance with many previous studies that showed positive effects of mindfulnessbased training on psychological well-being and quality of life (i.e., Baer 2003; Brown and Ryan 2003; Carlson and Brown 2005; Kabat-Zinn 1994; Kabat-Zinn et al. 1985; Segal et al. 2002).

Third, it was demonstrated that the MAAS-A correlated positively but not perfect with another very recently developed measure of mindfulness for children and adolescents, the CAMM. The MAAS-A and the CAMM are based on slightly different conceptualizations of mindfulness. The items of the CAMM are based on a multidimensional conceptualization of mindfulness. The content of the items refers to facets of observing, acting with awareness, and accepting without judgment (Greco et al. 2011). The MAAS-A items mainly focus on the attention and awareness of the present moment. In a previous version of the MAAS, items of a second component of mindfulness, 
namely acceptance, were included, but it was found that this acceptance factor did not explain any additional variance as compared to the present-centered attention and awareness factor (Brown and Ryan 2001).

Fourth, correlational analyses demonstrated a negative relationship, as expected, between mindfulness and rumination, catastrophizing, and stress. Mindfulness and rumination or catastrophizing seem to be opposite coping attitudes. Whereas rumination and catastrophizing are related to repeating negative thoughts about stressful situations, mindfulness is more related to nonjudgmentally observing and accepting these thoughts and stressors. Marks et al. (2010) found that the relationship between stressors in daily life and symptoms of anxiety and depression was positively mediated by mindfulness but negatively mediated by rumination. Thus, being more mindful lowers the anxious and depressed symptoms which can be caused by daily stressors. Mindfulness might be considered a protective factor. Further, the stress-reducing effects of mindfulness-based interventions has been shown by several studies (i.e., Baer 2003; Kabat-Zinn 1994; Hofmann et al. 2010), and therefore, the negative relation between a measure of mindfulness and a measure of stress is as expected. Interesting however is that not only is mindfulness as measured by the MAAS(-A) negatively related to selfreported stress but it is also shown that the so-called stress hormone cortisol might be a potential objective biomarker in evaluating the effectiveness of MBSR (Matousek et al. 2010) and cortisol levels decrease after participation in MBSR (Marcus et al. 2003).

Further, it was shown that mindfulness correlated positively as expected with happiness and healthy selfregulation. An open attention and awareness to experiences of the present moment is associated with feelings of well-being. This is in agreement with previous studies in which meditation and happiness were found to be related (i.e., Hanson and Mendius 2009). However, the expected positive relation between mindfulness and acceptance was not found. Moreover, the association was even in the opposite direction (non-significant) of what was expected. Since mindfulness includes acceptance of things as they are, non-judgmentally and in the present moment, we expected a positive relationship between the MAAS-A and a measure of acceptance. However, it seems that the MAAS-A specifically measures attention and awareness for daily situations, whereas the element of non-judgmental acceptance is not so much covered by items of the MAAS-A. Items from the CERQ seem to be mainly related to acceptance of events that have happened to you, whereas mindfulness refers more to acceptance of one's thoughts and feelings, good or bad. It could therefore be that the MAAS-A is not positively related to the
CERQ-acceptance items but does show this positive association to the CAMM items. Some items of the CAMM are related to (not) accepting one's thoughts, feelings, or emotions (i.e., "I push away thoughts that I don't like", "I think that some of my feelings are bad and that I shouldn't have them".) The CERQ seems to be focusing on coping styles in case of the occurrence of (negative) events, whereas mindfulness, or the CAMM or MAAS-A measurements, attempts to tap onto a state of awareness, a state of non-judgmental consciousness without any necessary relationship to the occurrence of actual events. Further, as was referred to by Jermann et al. (2006), items of passive resignation, which is considered a less adaptive strategy, and items of calm acceptance, which is considered an adaptive strategy, are combined in this one factor of acceptance. This makes this scale more difficult to interpret unambiguously. Combined with the low number of items on this subscale, these issues may be related to the unexpected findings. Another explanation could be that in community adolescents, being mindful does not necessarily imply an attitude of accepting without judgment. Adolescence is a time in which fast and black-andwhite judging is the norm. Therefore, correlations between the MAAS and acceptance may only appear in adults. Further research into the developmental course of the association between mindful awareness and acceptance without judging would be helpful to shed more light on this issue.

When adolescents with and without a chronic disorder such as asthma were compared, we found a higher level of mindfulness in the adolescents without a chronic disorder. This was as expected since it seems likely that when an adolescent often suffers from aches and pains, the focus of his/her attention will be directed to these pains. Due to regular sickness periods, it might be more difficult to observe and be aware of thoughts and feelings in an open, non-judgmental style. The adolescent's mind might simply be occupied with being sick more often than others around them. This seems in accordance with the demonstrated negative relationships between pain intensity, pain-related fear, and functional disabilities on the one hand and mindfulness on the other hand (Schütze et al. 2010). One could further speculate that the association between mindfulness and chronic somatic disorders may be bidirectional, that is, that being attentive and aware of physical symptoms and observing them as they come and go without judgment may help to overcome such symptoms. In line, mindfulness training has been found to be effective in adult patients with somatic disorders (i.e., Kabat-Zinn et al. 1985); however, studies assessing the effects of mindfulness training for adolescents with somatic disorders are to be awaited.

Last, contrary to our predictions, adolescents without meditation or yoga experience scored higher on mindfulness 
than those with meditation or yoga experience. This seems surprising but could be due to several factors. The sample of adolescents with meditation or yoga experience was very small $(10 \%)$, and meditation and yoga experience were not separately inquired. It could therefore be that these adolescents simply participated in regular yoga classes with no practice in meditation. However, our findings bear some resemblance to those of MacKillop and Anderson (2007) who found that in a sample of college students (of which also around $10 \%$ had meditation experience), the MAAS was unrelated to meditation experience. They concluded that novice level meditation, which is also applicable to our sample, is not necessarily associated with greater mindfulness. Relations between the MAAS and meditation were shown only in highly experienced Zen meditators (Brown and Ryan 2003). Another, related explanation is that beginning levels of mindfulness training make participants aware of how often they are mindless or not aware of the present moment. This awareness of mindlessness may affect the way they fill in the questionnaire, reducing their mindfulness scores, which may not necessarily reflect an actual lower state of mindfulness. A third explanation concerns the fact that those with mindfulness or yoga experience were self-selected. It could well be that adolescents who suffer from problems that are related to lack of mindfulness, such as high rumination, may look for mindfulness and yoga courses. In other words, the group with experience in mindfulness may have been the ones that needed it most, which is reflected in lower mindfulness scores. This hypothesis is strengthened by our findings on post hoc analyses that adolescents with meditation experience scored significantly higher on self-reported stress than adolescents without this experience, $t(715)=3.12$; $p=.002$, and adolescents with meditation experience reported a lower quality of life than adolescents without this experience, $t(676)=-3.14 ; p=.002$. Future research is needed to evaluate how mindfulness interventions affect MAAS-A scores.

A few limitations of the current study need to be mentioned. First, only higher educated adolescents of Dutch origin were included. Out of at least five different levels of high school in the Netherlands, only adolescents of the highest two levels were studied. Although this obviously bears limitations in the representativeness of the sample, it is in line with previous studies of mindfulness questionnaires where higher educated samples are included (i.e., Baer et al. 2006, 2008) and with the higher level of education which is commonly observed in meditation practitioners (Baer et al. 2008). In addition, we question whether items of the MAAS-A (or maybe from mindfulness questionnaires in general) are comprehensible for people with lower intellectual functioning since a relatively high level of reflection and verbal expression of one's inner state seems to be required.

Second, in our study, the MAAS-A was not administered to a clinical group like it was done in the original validation study by Brown et al. (2011). We can therefore draw no conclusions about the psychometric properties of the MAAS-A in adolescents with psychiatric symptoms or disorders. This will be a next step in our investigations. We did however see in our pilot study that mindfulness (as measured by the adult version of the MAAS) was significantly increased immediately after MBSR training but also at 8-week follow-up in a group of adolescents with externalizing disorders (Bögels et al. 2008). In line, we recently found significant direct improvements in mindful awareness (as measured with the MAAS) in parents of children with ADHD who participated in a mindful parenting training (Van der Oord et al. 2011). However, in future studies, the psychometric properties of the MAAS-A in clinical samples needs to be assessed.

Summarized, overall the Dutch version of the MAAS-A shows favorable psychometric properties which confirm the findings of the original USA-based MAAS-A. The Dutch version of the MAAS-A is reliable and consists of one factor in a sample of adolescents from the general community. In addition to the original MAAS-A, we found this one-factor structure and high internal consistency to be met also in the younger adolescents since our sample consisted of 11-17-year-olds. Negative correlations were found with constructs such as rumination, catastrophizing, and stress, and positive correlations, as expected, were found with happiness, healthy selfregulation, quality of life, and another measure for mindfulness assessment in children and adolescents. More surprising was that no association with acceptance was found which could be related to the different nature of the measures or might imply that being mindful in adolescence, a time in which fast black-and-white judgments are made, does not necessarily imply an attitude of accepting without judgment. Also interesting is that adolescents without meditation experience scored higher on the MAAS-A than adolescents with this experience. This might be related to the fact that the adolescents with meditation experience were also the ones who reported higher stress levels and a lower quality of life and therefore might have needed the meditation more than the others.

Open Access This article is distributed under the terms of the Creative Commons Attribution Noncommercial License which permits any noncommercial use, distribution, and reproduction in any medium, provided the original author(s) and source are credited. 


\section{References}

Baer, R. A. (2003). Mindfulness training as a clinical intervention: a conceptual and empirical review. Clinical Psychology: Science and Practice, 10, 125-143.

Baer, R. A., Smith, G. T., Hopkins, J., Krietemeyer, J., \& Toney, L. (2006). Using self-report assessment methods to explore facets of mindfulness. Assessment, 13, 27-45.

Baer, R. A., Smith, G. T., Lykins, E., Button, D., Krietemeyer, J., Sauer, S., et al. (2008). Construct validity of the Five Facet Mindfulness Questionnaire in meditating and non-meditating samples. Assessment, 15, 329-342.

Bastiaansen, D., Koot, H. M., Bongers, I. L., Varni, J. W., \& Verhulst, F. C. (2004). Measuring quality of life in children referred for psychiatric problems: psychometric properties of the PedsQLTM 4.0 generic core scales. Quality of Life Research, 13, 489-495.

Biegel, G. M., Brown, K. W., Shapiro, S. L., \& Schubert, C. M. (2009). Mindfulness-based stress reduction for the treatment of adolescent psychiatric outpatients: a randomized clinical trial. Journal of Consulting and Clinical Psychology, 5, 855866.

Bishop, S. R., Lau, M., Shapiro, S., Carlson, L., Anderson, N. C., Carmody, J., et al. (2004). Mindfulness: a proposed operational definition. Clinical Psychology: Science and Practice, 11, 230241.

Black, D. S., Milam, J., \& Sussman, S. (2009). Sitting meditation interventions among youth: a review of treatment efficacy. Pediatrics, 124, 532-541.

Bögels, S. M., Hoogstad, B., van Dun, L., de Schutter, S., \& Restifo, K. (2008). Mindfulness training for adolescents with externalizing disorders and their parents. Behavioral and Cognitive Psychotherapy, 36, 193-209.

Bootzin, R. R., \& Stevens, S. J. (2005). Adolescents, substance abuse, and the treatment of insomnia and daytime sleepiness. Clinical Psychology Review, 25, 629-644.

Brown, K. W., \& Cordon, S. L. (2009). Toward a phenomenology of mindfulness: subjective experience and emotional correlates. In F. Didonna (Ed.), Clinical handbook of mindfulness (pp. 59-81). New York, NY: Springer.

Brown, K. W., \& Ryan, R. M. (2001). Testing a two-factor model of mindfulness. Unpublished data. Rochester: University of Rochester.

Brown, K. W., \& Ryan, R. M. (2003). The benefits of being present: mindfulness and its role in psychological well-being. Journal of Personality and Social Psychology, 84, 822-848.

Brown, K. W., Ryan, R. M., \& Creswell, J. D. (2007). Mindfulness: theoretical foundations and evidence for its salutary effects. Psychological Inquiry, 18, 211-237.

Brown, K.W., West, A.M., Loverich, T.M., Biegel, G.M. (2011). Assessing adolescent mindfulness: validation of an adapted Mindful Attention Awareness Scale in adolescent normative and psychiatric populations. Psychological Assessment. doi:10.1037/ a0021338.

Burke, C. A. (2009). Mindfulness-based approaches with children and adolescents: a preliminary review of current research in an emerging field. Journal of Child and Family Studies, 19, 133144.

Carlson, L. E., \& Brown, K. W. (2005). Validation of the mindful attention awareness scale in a cancer population. Journal of Psychosomatic Research, 58, 29-33.

Davis, T. E., May, A., \& Whiting, S. A. (2011). Evidence-based treatment of anxiety and phobia in children and adolescents: current status and effects on the emotional response. Clinical Psychology Review, 31, 592-602.
Garnefski, N., Kraaij, V., \& Spinhoven, Ph. (2001). Negative life events, cognitive emotion regulation and depression. Personality and Individual Differences, 30, 1311-1327.

Greco, L., \& Hayes, S. C. (2008). Acceptance and mindfulness treatments for children and adolescents: A practitioners guide. Oakland: New Harbinger.

Greco, LA., Baer, RA., Smith, GT. (2011). Assessing mindfulness in children and adolescents: development and validation of the Child and Adolescent Mindfulness Measure (CAMM). Psychological Assessment. doi:10.1037/a0022819.

Grossman, P., Niemann, L., Schmidt, S., \& Walach, H. (2004). Mindfulness-based stress reduction and health benefits: a meta-analysis. Journal of Psychosomatic Research, 57, 35-43.

Hair, J. F., Anderson, R. E., Tatham, R. L., \& Black, W. C. (1995). Multivariate data analysis (4th ed.). Englewood Cliffs: Prentice-Hall.

Hanson, R., \& Mendius, R. (2009). Buddhaś brain. The practical neuroscience of happiness, love and wisdom. Oakland: New Harbinger.

Hartong, I. M., Krol, M., Maaskant, A., Te Plate, A., \& Schuszler, D. (2003). Stress Vragenlijst voor Kinderen (SVK). Internal report. Amsterdam: University of Amsterdam.

Hofmann, S. G., Sawyer, A. T., Witt, A. A., \& Oh, D. (2010). The effect of mindfulness-based therapy on anxiety and depression: a meta-analytic review. Journal of Consulting and Clinical Psychology, 78, 169-183.

Jermann, F., Van der Linden, M., d'Acremont, M., \& Zermatten, A. (2006). Cognitive Emotion Regulation Questionnaire (CERQ). Confirmatory factor analysis and psychometric properties of the French translation. European Journal of Psychological Assessment, 22, 126-131.

Jha, A. P., Krompinger, J., \& Baime, M. J. (2007). Mindfulness training modifies subsystems of attention. Cognitive, Affective, \& Behavioral Neuroscience, 7, 109-119.

Kabat-Zinn, J. (1994). Wherever you go, there you are: Mindfulness meditation in everyday life. New York: Hyperion.

Kabat-Zinn, J., Lipworth, L., \& Burney, R. (1985). The clinical use of mindfulness meditation for the self-regulation of chronic pain. Journal of Behavioral Medicine, 8, 163-190.

Lyubomirsky, S., \& Lepper, H. S. (1999). A measure of subjective happiness: preliminary reliability and construct validation. Social Indicators Research, 46, 137-155.

MacKillop, J., \& Anderson, E. J. (2007). Further psychometric validation of the mindful attention awareness scale (MAAS). Journal of Psychopathology and Behavioral Assessment, 29, 289-293.

Marcus, M. T., Fine, P. M., Moeller, F. G., Khan, M. M., Pitts, K., Swank, P. R., et al. (2003). Change in stress levels following mindfulness-based stress reduction in a therapeutic community. Addictive Disorders \& Their Treatment, 2, 63-68.

Marks, A. D. G., Sobanski, D. J., \& Hine, D. W. (2010). Do dispositional rumination and/or mindfulness moderate the relationship between life hassles and psychological dysfunction in adolescents? The Australian and New Zealand Journal of Psychiatry, 44, 831-838.

Matousek, R. H., Dobkin, P. L., \& Pruessner, J. (2010). Cortisol as a marker for Improvement in mindfulness-based stress reduction. Complementary therapies in clinical practice, 1, 13-19.

Meijer, A. M., van Oostveen, S. J. E., \& Stams, G. J. J. M. (2008). Zorgen voor een zieke ouder: De relatie tussen mantelzorg, ziekte van de ouder en gedragsproblemen bij kinderen. Kind en Adolescent, 29, 208-220.

Merikangas, K. R., He, J., Burstein, M., Swanson, S. A., Avenevoli, S., Cui, L., et al. (2010). Lifetime prevalence of mental disorders in U.S. adolescents: results from the National Comorbidity Study-Adolescent Supplement (NCS-A). Journal of the American Academy of Child and Adolescent Psychiatry, 49, 980-989. 
NIMH. (2006). Agency for Healthcare Research and Quality. Medical expenditure panel survey. Bethesda: National Institute of Mental Health.

Pelham, W. E., \& Fabiano, G. A. (2008). Evidence-based psychosocial treatments for attention-deficit/hyperactivity disorder. Journal of Clinical Child and Adolescent Psychology, 37, 184-214.

Poos, M. J. J. C., Smit, J. M., Groen, J., Kommer, G. J., \& Slobbe, L. C. J. (2008). Kosten van ziekten in Nederland 2005. RIVM rapport $n r$ 270751019. Bilthoven: RIVM.

Schroevers, M., Nyklíček, I., \& Topman, R. (2008). Validatie van de Nederlandse versie van de Mindful Attention Awareness Scale (MAAS). Gedragstherapie, 41, 225-240.

Schütze, R., Rees, C., Preece, M., \& Schütze, M. (2010). Low mindfulness predicts pain catastrophizing in a fear-avoidance model of chronic pain. Pain, 148, 120-127.

Segal, Z., Williams, J., \& Teasdale, J. (2002). Mindfulness-based cognitive therapy for depression. A new approach to preventing relapse. New York: The Guilford Press.

Singh, N. N., Lancioni, G. E., Singh Joy, S. D., Winton, A. S. W., Sabaawi, M., Wahler, R. G., et al. (2007). Adolescents with conduct disorder can be mindful of their aggressive behavior. Journal of Emotional and Behavioral Disorders, 15, 65-63.

Tkach, C., \& Lyubomirsky, S. (2006). How do people pursue happiness?: Relating personality, happiness-increasing strategies, and well-being. Journal of Happiness Studies, 7, 183-225.

Van de Weijer-Bergsma, E., Formsma, A. F., de Bruin, E. I., \& Bögels, S. M. (2011). Effectiveness of mindfulness-based CBT on behavioral symptoms and attention performance in adolescents with attention deficit hyperactivity disorder. Submitted.

Van der Oord, S., Bögels, S. M., \& Peijnenburg, D. (2011). The effectiveness of mindfulness training for children with ADHD and mindful parenting for their parents. Journal of Child and Family Studies, 19, 157-174.

Varni, J. W., Seid, M., \& Rode, C. A. (1999). The PedsQL ${ }^{\mathrm{Tm}}$ : measurement model for the pediatric quality of life inventory. Medical Care, 37, 126-139.

Varni, J. W., Seid, M., \& Kurtin, P. S. (2001). PedsQL(tm) 4.0: reliability and validity of the Pediatric Quality of Life Inventory (tm) Version 4.0 Generic Core Scales in healthy and patient populations. Medical Care, 39, 800-812.

Watanabe, N., Hunot, V., Omori, I. M., Churchill, R., \& Furukawa, T. A. (2007). Psychotherapy for depression among children and adolescents: a systematic review. Acta Psychiatrica Scandinavica, $116,84-95$.

Weisz, J. R., McCarty, C. A., \& Valeri, S. M. (2006). Effects of psychotherapy for depression in children and adolescents: a meta-analysis. Psychological Bulletin, 132, 132-149.

West, A. M. (2008). Mindfulness and the well-being in adolescence: an exploration of four mindfulness measures with an adolescent sample. Unpublished doctoral dissertation, Central Michigan University.

West, A. M., \& Kram, G. (2008). Mindfulness program evaluation in an adjudicated juvenile sample. Unpublished manuscript, Central Michigan University.

West, A. M., Penix-Sbraga, T., \& Poole, D. A. (2008). Measuring mindfulness in youth: development of the Mindful Thinking and Action Scale for adolescents. Unpublished manuscript, Central Michigan University.

Zylowska, L., Ackerman, D. L., Yang, M. H., Futrell, J. L., Horton, N. L., Hale, T. S., et al. (2008). Mindfulness meditation training in adults and adolescents with ADHD: a feasibility study. Journal of Attention Disorders, 11, 737-746. 